\title{
DIFICULDADES DOS ESTUDANTES DE ENFERMAGEM NA APRENDIZAGEM DO DIAGNÓSTICO DE ENFERMAGEM, NA PERSPECTIVA DA METACOGNIÇÃO
}

Nursing students' difficulties in the learning of diagnostics in nursing, in a metacognitive perspective

Dificultades de los estudiantes de enfermería e aprendizaje de diagnósticos de enfermería, en la respectiva de la meta cognición

Ana Gracinda Ignácio da Silva ${ }^{1}$
Márcia de Assunção Ferreira ${ }^{4}$

Mauricio Abreu Pinto Peixoto²

Jaqueline Santos de Andrade Martins ${ }^{5}$
Marcos Antonio Gomes Brandão ${ }^{3}$

\section{RESUMO}

0 objetivo geral é investigar a aprendizagem do diagnóstico em enfermagem na área hospitalar, de alunos concluintes do curso de graduação, na perspectiva metacognitiva. De natureza qualitativa, desenvolveram-se entrevistas individuais com dezenove alunos da quinta série, do último estágio supervisionado na área hospitalar. A análise temática de conteúdo indicou dificuldades dos alunos no processo de aprendizagem, relacionadas à estrutura teórica, à aplicação prática de diagnosticar, ao conhecimento e ao uso das classificações diagnósticas, faltando-lhes a percepção do raciocínio clínico envolvido e que resulta no diagnóstico. Acredita-se que a metacognição possa ser eficaz no controle das dificuldades de aprendizagem dos alunos, por incluir o conhecimento de estratégias de regulação de desempenho, de planejamento e controle do processo de aprendizagem.

Palavras-chave: Aprendizagem. Diagnóstico de Enfermagem. Cognição

\section{Abstract}

The general objective is to investigate, in a metacognitive perspective, the learning of diagnostics with undergraduate students in a hospital nursing area. Of qualitative nature, the scientific phenomenological method was applied through individual interviews with nineteen students from the fifth year, the last supervised year of hospital area internship. The theme analysis of content indicated that the students had difficulties in their learning processes related to theoretical structure, practical diagnostic application, knowledge and use of diagnostic classifications, and lack of perception of the clinical thinking and results involved in diagnostics. Concluding that metacognition, including knowledge of regulation strategies of performance and the planning and control of the learning process, may be effective in controlling the difficulties in the students' learning.

Keywords: Learning. Nursing Diagnostics. Cognition

\section{Resumen}

El objetivo general es investigar, en la perspectiva meta cognitiva, el aprendizaje del diagnóstico en enfermería en el área hospitalaria, de alumnos concluyentes del curso de graduación. De naturaleza cualitativa, se aplicó el método fenomenológico científico a través de entrevistas individuales con diecinueve alumnos de la quinta serie, del último período de prácticas supervisadas en el área hospitalaria. El análisis temático de contenido indicó dificultades de los alumnos en el proceso de aprendizaje, relacionadas a la estructura teórica, la aplicación práctica de diagnosticar, al conocimiento y uso de las clasificaciones diagnósticas, les faltando la percepción de que esto envuelve raciocinio clínico y que resulta en el diagnóstico. Se concluye que la meta cognición, por incluir el conocimiento de estrategias de regulación de desempeño, de planificación y control del proceso de aprendizaje, pueda ser eficaz en el control de las dificultades de aprendizaje de los alumnos.

Palabras claves: Aprendizaje. Diagnóstico de Enfermería. Cognición

\footnotetext{
${ }_{1}^{1}$ Mestre em Enfermagem. Doutora pela Escola de Enfermagem Anna Nery/ UFRJ. Professora Adjunto do Departamento de Enfermagem Hospitalar da Universidade do Estado do Pará (UEPA). Membro do Núcleo de Pesquisa de Fundamentos do Cuidado de Enfermagem (Nuclearte). Membro do Grupo de Estudos em Aprendizagem e Cognição (GEAC). Bolsista do CNPq. Belém-PA. Brasil. E-mail: anagracinda@terra.com.br; ${ }^{2}$ Doutor em Medicina pela Faculdade de Medicina da UFRJ. Professor Adjunto e Pesquisador do Laboratório de Currículo e Ensino (LCE/NUTES). Líder do Grupo de Estudos em Aprendizagem e Cognição (GEAC). Orientador do doutorado. Rio de Janeiro - RJ. Brasil. E-mail: mpeixoto@nutes.ufrj.br; ${ }^{3}$ Doutor em Enfermagem. Professor Adjunto do Departamento de Enfermagem Fundamental da Escola de Enfermagem Anna Nery / UFRJ. Pesquisador Permanente do Grupo de Estudos em Aprendizagem e Cognição (GEAC). Rio de Janeiro - RJ. Brasil. E-mail: marcosagbrandao@yahoo.com.br; ${ }^{4}$ Doutora em Enfermagem. Professora Titular do Departamento de Enfermagem Fundamental da Escola de Enfermagem Anna Nery/UFRJ. Pesquisadora do CNPq. Membro da Diretoria do Nuclearte. Rio de Janeiro - RJ. Brasil. E-mail: marciadeaf@ibest.com.br; ${ }^{5}$ Mestre em Enfermagem. Doutoranda pela Escola de Enfermagem Anna Nery/UFRJ. Membro do Núcleo de Pesquisa em Fundamentos do Cuidado de Enfermagem (Nuclearte). Membro do Grupo de Estudos em Aprendizagem e Cognição (GEAC). Bolsista pela CAPES. Rio de Janeiro - RJ. Brasil. E-mail: jaquelinedeandrademartins@gmail.com
} 


\section{INTRODUÇÃO}

0 diagnóstico é a segunda etapa do processo de enfermagem, e por meio dele se concretiza a interpretação clínica de dados coletados dos pacientes, tendo por base evidencias científicas. Objetiva orientar o planejamento, a realização e a avaliação do cuidado de enfermagem. ${ }^{1}$

0 uso do termo "diagnóstico de enfermagem"::30 confirmou os enfermeiros como diagnosticadores em sua prática, potencializando suas habilidade de decidir sobre 0 cuidado de enfermagem. Assim, os diagnósticos de enfermagem são a base do cuidado. Desse modo, os enfermeiros precisam desenvolver competências para diagnosticar o que incluem habilidades intelectuais e processos mentais em conhecimentos diagnósticos e uso desses conhecimentos respectivamente.

0 processo cognitivo de elaboração dos diagnósticos, que é comumente denominado raciocínio diagnóstico, exige de alunos e profissionais de enfermagem competência de nível elevado. Esse dado não se trata de atividade corriqueira, o aprendizado do diagnóstico requer monitoramento e controle do pensamento por meio da metacognição. ${ }^{1}$

A metacognição abrange a monitoração do sistema cognitivo e emocional. Foi termo introduzido por Flavel $^{2} \mathrm{em}$ 1970 para definir conhecimento sobre os próprios processos e produtos cognitivos. Nessa perspectiva depreende-se a competência do indivíduo que precisa refletir sobre seus processos mentais. ${ }^{2}$

Atividades de maior dificuldade, complexidade e com certo grau de ineditismo costumam requerer do indivíduo a aplicação do processo de reflexão, o que não costuma acontecer em processos repetitivos, de reprodução de ações automatizáveis e extremamente familiares. 0 aprendizado diagnóstico inicia-se na graduação e percorre a vida profissional do enfermeiro; contudo, jamais pode ser considerado uma atividade fácil, simples e plenamente repetitiva.

Estudos têm mostrado dificuldades de aprendizagem do diagnóstico de enfermagem, dentre elas: deficiência de aprofundamento de conhecimentos para diagnosticar; currículo que não contempla conteúdos necessários ao desenvolvimento do tema de forma contínua; resistência à proposta dos diagnósticos; dificuldades em coletar dados, em realizar o enunciado diagnóstico, em identificar as causas que fundamentam o diagnóstico de enfermagem; o processo mental envolvido; dificuldade do estudante de fazer relações significativas com conteúdos anteriores e fazer interpretações e julgamentos que conduzam a solução de problemas. Há falta de conhecimento e experiência, falta de consciência das limitações e passos para realizar o diagnóstico de enfermagem, definida como falta de controle do pensamento, o que dificulta a consciência do funcionamento da mente, das falhas cometidas e de como se qualifica o próprio pensar. ${ }^{3-5}$

Presume-se que algumas das dificuldades apresentadas exijam melhor investigação, em especial, a partir de uma perspectiva interpretativa com base metacognitivista.
Há que se indicar que a estrutura de diagnóstico de enfermagem utilizada pelos estudantes é a proposta pela North American Nursing Diagnosis Association (NANDA) e que implica basicamente três elementos: categoria diagnóstica (o termo diagnóstico), características definidoras (sinais e sintomas) e fatores relacionados (etiologias) ou fatores de risco (para situaç̃̃es de desenvolvimento potencial).

A metacognição envolve duas importantes funções: a de monitoração e controle. E envolve três importantes estágios em relação à aprendizagem: aquisição, retenção e recuperação. ${ }^{6}$

0 uso da metacognição inclui planejar, direcionar e avaliar seu comportamento, alocar estratégias de aprendizagem para resolver problemas e monitorizar seu sucesso ou seu fracasso no desempenho, sendo capaz de decidir sobre uso de estratégias diferentes e saber do esforço necessário para usar essas estratégias. 0 conhecimento das estratégias envolve tomar consciência das semelhanças e diferenças entre elas, e de quando e onde deve ser usada cada uma delas.?

No processo de aprendizagem, a metacognição traz muitos benefícios; dentre outros, pode determinar o modo de o aluno melhorar o seu desempenho e organizar seu pensamento e, ainda, prevê o grau de aprendizagem de determinado tópico.?

Partindo dos benefícios de uma abordagem de investigação metacognitivista, este estudo tem como objeto a Aprendizagem do Diagnóstico de Enfermagem por alunos concluintes do curso de graduação em enfermagem, com objetivo de investigar, na perspectiva metacognitiva, as dificuldades dos alunos em seu processo de aprendizagem do diagnóstico de enfermagem na área hospitalar.

\section{MÉTODO}

Estudo de natureza qualitativa e descritiva, que teve como contexto o Curso de Graduação em Enfermagem de uma instituição pública de ensino superior, no município de BelémPará. Os sujeitos participantes da pesquisa foram 19 alunos matriculados na quinta série deste curso dos 22 que cursavam o último estágio supervisionado na área hospitalar. A escolha dos participantes deu-se sob a forma de censo. Nominadas neste texto, as citações o foram com nomes fictícios, de modo a preservar 0 anonimato.

A técnica de produção de dados foi entrevista orientada por um roteiro semiestruturado registrada em formato digital. Como técnica complementar, utilizou-se o diário clínico com o objetivo de descrever as ações clínicas. Esta técnica é uma extensão do diário de aula, ${ }^{8}$ que é uma técnica de documentação, utilizada para registro de informações para análise, descrições e reflexões sobre fatos vivenciados. 0 período de produção dos dados abrangeu de agosto a novembro de 2009.

Os dados foram armazenados no computador no programa Voice Editing e então reproduzidos e transcritos na íntegra para análise. Após a transcrição, todas as entrevistas foram validadas pelos sujeitos que as concederam. 
Foi realizada a análise temática tendo por bases variáveis empíricas identificadas a partir de unidades de significação. ${ }^{9}$ Todo o corpus foi sistematicamente disposto em planilhas no Programa Excel, servindo de base para a análise temática.

0 projeto foi aprovado pelo Comitê de Ética em Pesquisa, 26/05/2009, protocolo no 22/09, com assinatura do termo de consentimento livre e esclarecido pelos sujeitos, cujo anonimato foi mantido por identificação em pseudônimo.

\section{APRESENTAÇÃO E DISCUSSÃO DE RESULTADOS}

Os alunos entrevistados estavam na faixa etária de 21 a 52 anos de idade, mas, preponderantemente, com 22-23 anos de idade ( 10 sujeitos). Entretanto, as demais faixas acima de 23 anos ( 6 alunos) e abaixo de 22 anos ( 2 sujeitos) indicam um grupo com características de geração semelhante, contudo, divergindo em virtude da experiência individual. Em relação ao gênero, os entrevistados foram cinco homens e 14 mulheres; entre esses, somente dois alunos são independentes financeiramente de pais ou maridos. Treze desses alunos estudaram em escola particular.

A partir da organização e análise de conteúdo temática das entrevistas, foi identificada a temática "Aprendizagem", que indicou a subcategoria denominada "dificuldades de aprendizagem do diagnóstico de enfermagem", que emergiu do material empírico, é foco deste estudo e será apresentada a seguir.

\section{Aprendizagem diagnóstica: Dificuldades}

Dificuldades na aprendizagem do diagnóstico de enfermagem foram informadas por 12 doze alunos; quatro negaram sua ocorrência e três tiveram dúvidas em suas assertivas. À luz dos resultados, aprender a fazer diagnóstico de enfermagem foi considerada tarefa difícil para a maioria dos alunos pesquisados por diversos motivos e em diferentes níveis de dificuldade.

Foram várias as dificuldades apontadas no processo de aprendizagem diagnóstica. Essas dificuldades estão relacionadas a duas dimensões importantes no processo de aprendizagem: a) a deficiência de embasamento teórico necessário para fundamentar e desenvolver conhecimento teórico e prático que facilite a habilidade de diagnosticar, e b) o processo mental necessário para organização e uso do conhecimento diagnóstico.

\section{a)Embasamento teórico}

Nesta dimensão estão inclusas dificuldades relacionadas ao manuseio e entendimento dos manuais (NANDA e Carpenito). Foram relatadas as dificuldades em: 1) redigir o diagnóstico seguindo modelos existentes (NANDA e
Carpenito) em suas partes componentes; 2) fazer diagnósticos sem depender exclusivamente do livro; 3 ) conhecer a doença e termos técnicos diagnósticos; 4) fazer anamnese completa; 5) discriminar rótulos diagnósticos semelhantes e 6) processar a quantidade excessiva de informação.

Reconhecidamente, o aprender é diferente de ensinar, porque aprender é um processo que acontece no aluno, e este é 0 agente essencial. ${ }^{10}$ Isso pode justificar a importância da aprendizagem da habilidade intelectual, que inclui 0 conhecimento sobre o diagnóstico de enfermagem. ${ }^{1}$ A deficiência nessa habilidade correlaciona-se com as dificuldades relatadas pelos sujeitos.

Uma dificuldade de todos os alunos, nesta dimensão, é a redação diagnóstica; isto é, expressar por escrito e no formato exigido pela taxonomia diagnóstica, o que foi observado durante o processo de coleta de dados. Da prática, verifica-se que tanto os enfermeiros como os acadêmicos identificam com relativa facilidade os problemas do cliente durante a sua assistência. No entanto, é difícil para eles formalizar por escrito o enunciado do diagnóstico.

0 diagnóstico de enfermagem constitui-se de três partes: o rótulo diagnóstico, a causa e suas características definidoras (evidências) conforme a estrutura da NANDA. A dificuldade para os alunos está em expressá-los nas suas partes componentes. Para tal, eles afirmam primeiro ser necessário memorizar os rótulos diagnósticos e suas partes associadas. Em seguida, relacionar o que foi memorizado com o que foi observado na prática. E neste sentido, a grande quantidade de rótulos torna seu aprendizado difícil.

Finalmente, tendo sido atribuído um dado rótulo, é necessário então adicionar as características definidoras e a causa. Aumenta a dificuldade quando o problema do cliente permite a consideração de variados rótulos semelhante. Como nos outros, mas, especialmente neste caso, torna-se necessário identificar com precisão a característica definidora, gerando assim uma dificuldade adicional.

Parece haver, neste caso, dois aspectos causais, pelo menos do ponto de vista metacognitivo. Em primeiro lugar percebe-se sua dificuldade em compreender a estruturação de um diagnóstico. 0 aluno tenta memorizar problemas, rótulos, características e causas como se estas não fossem partes de um todo orgânico. Neste sentido, então, aumenta erradamente seu esforço ao tentar memorizar informações desconectadas. Em segundo lugar e como consequência disto, cria um "abismo epistemológico" entre a taxonomia da NANDA, neste caso, e a realidade. É como se a assistência fizesse parte de um mundo diverso daquele presente no manual. Ao transferir para a assistência, esta falta de conexão "vê" (erradamente) um mundo desconectado, tornando difícil para ele relacionar eventos deste aspecto da realidade. Fica difícil atribuir a eventos e percepções diversas os significados de "causa" e "consequência" descritos nos manuais diagnósticos. 


\section{Dificuldades na apredizagem do diagnóstico de enfermagem}

Os alunos relatam ainda dificuldades em compreender o vocabulário usado nos manuais. Assim, o aluno, tendo nominado com termos de uso comum um dado problema, não consegue perceber porque não pode usá-los como tal, tendo de expressar-se com os termos mais técnicos usados na taxonomia. Como afirma Luz:

se o paciente apresenta úlcera de pressão, o diagnóstico seria úlcera de pressão, mas geralmente, na Carpenito, tem integridade da pele prejudicada, vai rebuscando os termos, eu sou um pouco mais direta.

Há aqui, assim nos parece, uma incompreensão epistemológica. 0 diagnóstico, "integridade tissular prejudicada" "1" não é sinônimo de "úlcera de pele" vez que esta é apenas uma de suas evidências. 0 rótulo em tela adiciona à evidência apresentada toda uma rede de conceitos e conhecimentos, fato não percebido pela aluna. Neste sentido então, apresentar este rótulo diagnóstico é revelar uma compreensão bem mais ampla do que o que se associa ao mero relato do observado.

Os alunos relatam também, um sentimento de insegurança, como um sentimento que reflete as dificuldades cognitivas de aprender diagnosticar. É relevante dizer que, concomitante às mudanças cognitivas, ocorrem processos emotivos no aluno gerando sentimentos positivos ou negativos que acompanham o processo de perceber e entender. Sentimentos que fazem parte do processo de aprender e refletem na maior capacidade de aprender, de manejar ou controlar a própria aprendizagem. ${ }^{10}$

Essas dificuldades levam a refletir que o processo de cuidar de enfermagem é uma ação profissional que ocorre em interação direta, por meio de atividades "para" e "com" o paciente e está relacionada ao conhecimento científico, habilidade, intuição, pensamento crítico e criatividade. ${ }^{12}$ Estas atividades precisam ser aprendidas e desenvolvidas durante a formação profissional do enfermeiro.

Existem ainda alguns aspectos que podem contribuir para essas dificuldades e que estão relacionadas à seleção de atividades de ensino-aprendizagem. 0 conteúdo informa e os métodos formam, e, assim, o tipo de atividade forma mais do que o conteúdo em si. ${ }^{10}$ Além disso, do ponto de vista metacognitivo, o domínio do conhecimento de si mesmo em relação ao conhecimento que possui, resulta em domínio de táticas de estudo, pois a metacognição pode beneficiar o estudo. Isso significa que a automonitoração da aprendizagem pode reduzir discrepâncias reveladas pela metacognição; entretanto, a monitoração metacognitiva é comprometida quando uma tarefa é ambígua. ${ }^{13}$

Construir um Diagnóstico de Enfermagem demanda a realização correta e completa da anamnese. Isso porque, não fazer de forma adequada, segundo eles, resulta na falta de dados que podem ajudar a fazer o diagnóstico. Esta parece ser mais uma dificuldade, e, sendo os diagnósticos de enfermagem a base para o atendimento, os enfermeiros precisam desenvolver competências para diagnosticar, entre essas, a competência técnica que é fundamental para realizar levantamentos de dados que sejam válidos e confiáveis. 0 levantamento de dados nesse caso é uma investigação de enfermagem "quando obtém dados necessários ao atendimento de enfermagem". 1:33

Outro aspecto a considerar no elenco de dificuldades para a aprendizagem diagnóstica é a falta dessa atividade na prática, porque os alunos relatam que ficam muito ocupados realizando procedimentos, e, por mais que estejam vendo e até pensem no diagnóstico que possivelmente o paciente tem, não fazem e não registram e, por isso, não aprendem, não desenvolvem conhecimento e habilidade. Esta dificuldade foi descrita pela maioria dos alunos, principalmente, nos diários clínicos elaborados por eles. Vilma, uma das alunas, relata:

A importância é dada em aprender procedimentos, e porque não dar importância também em aprender o diagnóstico? Quando se está fazendo o procedimento porque não questionar com a gente sobre o DE? É só, monitora o paciente, faz evolução, acompanha, faz exame físico, não se pede o diagnóstico, por quê? [...], nosso trabalho acaba se confundindo com o do técnico. (Vilma)

Sobre isso, os alunos percebem que eles fazem as atividades do técnico de enfermagem e, sendo assim, eles tem que executar procedimentos que já estão ordenados, principalmente nas prescrições médicas. Isso, entretanto, pode ser diferente, se o aluno cuidar de uma perspectiva diferente, ou seja, diagnosticando e utilizando seu vocabulário próprio para definir eventos de sua responsabilidade terapêutica. Nesta perspectiva, o aluno deixa de se ver como cumpridor de tarefas, e sim como um profissional incluído no compromisso social do cuidado de enfermagem, desenvolvendo, principalmente, ações específicas e que demandam da sua autonomia de prática profissional.

Em uma das unidades de registro analisadas encontrase a afirmação de que o diagnóstico é um conhecimento novo, e o que é novo assusta. Soma-se a isso que, nos hospitais, nem todos os enfermeiros fazem diagnósticos, além de não ser um conteúdo dado no início do curso, e isto também é tido como um aspecto que dificulta a aprendizagem diagnóstica. Neste sentido, quando os alunos se encontram em setores onde a enfermeira cuida a partir do diagnóstico de enfermagem, eles se engajam nessa ação com mais facilidade, o que reforça a importância dos ambientes de aprendizagens.

Nesse caso, cabe lembrar que diante da realidade descrita, a metacognição pode auxiliar o educador a construir ambientes de ensino que se focalizam na aprendizagem estratégica, flexível e criativa, na consciência das estratégias 
eficazes, na discussão sobre aspectos motivacionais e cognitivos do pensar. ${ }^{2}$

Além do embasamento teórico, os alunos entrevistados descreveram dificuldades que se relacionam com o processo de pensamento que leva a ação diagnóstica, como falaremos a seguir.

\section{b)Processo de Pensamento}

Nesta dimensão estão agrupadas dificuldades relacionadas a: 1) dúvidas sobre o que investigar; 2) não saber o que fazer com os dados do paciente 3) não saber discernir se é diagnóstico médico ou diagnóstico de enfermagem e 4) como chegar ao diagnóstico.

Diante do paciente, o aluno se diz não ter clareza do que deve investigar para subsidiar o diagnóstico de enfermagem; essa dificuldade leva a outro aspecto, que é o que fazer com esses dados. Neste caso, há uma dificuldade evidente de percepção pelo aluno da ação diagnóstica como consequência, ou resultado natural da avaliação e evolução do paciente e que ele, obrigatoriamente, realiza. Há dificuldade de tomar consciência do processo de pensamento envolvido na aplicação do conhecimento diagnóstico.

Parece haver uma percepção de que a avaliação que realiza com o paciente e o diagnóstico de enfermagem são coisas separadas, como se houvesse uma cisão completa entre a vida da prática e o manual diagnóstico, ao passo que, na anamnese de enfermagem, histórico, evolução ou qualquer registro da avaliação clínica do paciente estarão contempladas as características definidoras e fatores relacionados ou de riscos que vão resultar em um diagnóstico.

Esta dificuldade pode ocorrer porque o diagnóstico de respostas humanas é um processo complexo e necessita que 0 profissional seja capaz de interpretar o comportamento humano em relação à saúde. Isso ocorre em um profundo processo de interação de processos interpessoais, técnicos e intelectuais. 0 enfermeiro deve reconhecer as evidências e relacionar essa evidência com o conhecimento armazenado em sua memória, gerando significados. Este é um processo de pensamento ativo ${ }^{14}$ que leva o profissional a elaborar explicaç̃̃es possíveis para os dados e, neste caso, inicia o processo diagnóstico.

É importante mencionar que os estudiosos da memória ${ }^{2}$ distinguem as atividades de armazenamento das de recuperação; as primeiras colocam a informação na memória, enquanto a segunda as resgata. A recuperação do conhecimento armazenado significa reconhecer recordar e reconstruir esse conhecimento. 0 armazenamento e a recuperação na prática são interdependentes, e isso depende de alguns aspectos, como a forma de organização da informação, quando ela é armazenada, quais as pistas de recuperação e com que sucesso ela será recuperada posteriormente. ${ }^{2}$

Existem dois tipos de recuperação da memória: 0 reconhecimento e a recordação. A pessoa pode reconhecer algo como familiar, semelhante a algo experimentado anteriormente, um tipo de representação na memória, enquanto que recordar é um processo de recuperar a representação armazenada na memória. Na recordação, o trabalho de recuperação é maior e mais individual. Assim, após reconhecer algo como familiar, procede-se à recordação de informações adicionais sobre ela. ${ }^{2}$

Não saber discriminar se o problema evidenciado é compatível com diagnóstico médico ou de enfermagem significa que, do ponto de vista cognitivo, o diagnóstico de enfermagem é algo invisível, porque está oculto pelo diagnóstico médico. Isso quer dizer que o aluno de enfermagem aprende e valoriza sinais e sintomas relacionados à entidade nosológica médica em detrimento daqueles que caracterizam a prática de enfermagem.

Não saber como chegar ao diagnóstico de enfermagem está relacionado ao raciocínio envolvido no processo diagnosticar. 0 raciocínio inclui a percepção de autoeficácia, que se relaciona com as crenças pessoais do aluno, na sua capacidade de se organizar para efetuar os atos requeridos para conseguir uma realização. As fontes de informação para a percepção da autoeficácia estão nas experiências, na persuasão verbal e estado afetivo ou fisiológico. Esses elementos são imprescindíveis ao contexto do uso do processo diagnóstico na aprendizagem clínica. ${ }^{14}$

Neste aspecto, o controle e a monitoração do processo cognitivo diagnóstico são fundamentais para a compreensão do processo de pensamento envolvido no raciocínio diagnóstico. A monitoração faz com que o aluno use o conhecimento e as relações necessárias ao julgamento clínico que produza 0 diagnóstico. 0 processo de informação sobre o objeto e suas relações faz com que o aluno tome consciência do raciocínio envolvido e controle sobre sua aprendizagem diagnóstica. Este controle pode modificar seu comportamento em relação ao processo de raciocínio diagnóstico. ${ }^{14}$

Monitorar seu aprendizado envolve julgar seu conhecimento e ter confiança em recuperar suas respostas. 0 controle envolve aspectos como alocação de tempo de estudo, seleção de estratégias e finalização do processo de estudo. ${ }^{2}$ Os alunos pesquisados apresentaram aspectos de monitoração, já que souberam julgar suas limitações e potenciais como diagnosticadores; porém, eles relataram dificuldades em relação ao resgate de conhecimentos e relações necessárias para empreender o processo de raciocínio diagnóstico, o que leva a crer que não possuem controle sobre essa aprendizagem.

Para formular o diagnóstico segundo esses alunos, é preciso pensar de forma mais abrangente: no problema e no texto diagnóstico, relacionando-o com fatores causais e com os aspectos que evidenciam aquele diagnóstico.

É um processo que exige muito do aluno em termos de tempo de pensamento. Esse tempo de pensamento consiste na sistematização do processo e inclui desde a coleta de dados, o problema identificado até chegar ao diagnóstico na sua "redação com toda essa estrutura", e isto requer muito tempo. 
Neste ínterim, o tempo emerge como um fator importante porque com tempo se pode pensar melhor; se não tem tanto tempo, acaba-se perdendo uma questão, o insight que a fisiologia pode dar. Entretanto, a dinâmica do hospital requer que as atividades sejam feitas de forma rápida, incluindose aí as atividades afeitas ao diagnóstico. Isso faz com que o aluno primeiro escreva para depois pensar sobre o que escreveu, prejudicando o estabelecimento de relações que poderiam ter sido feitas para explicar algo, se houvesse mais tempo para isso.

A partir de processos mentais eficientes, o aluno poderá reconstruir as informações sobre o diagnóstico e que são novas para ele, descobrindo inter-relações que podem facilitar sua aprendizagem, identificar a origem de suas dificuldades e como superá-las, em um processo ativo de aprender a diagnosticar.

\section{CONSIDERAÇÕES FINAIS}

Investigar sobre a aprendizagem diagnóstica em enfermagem mostrou que muitas são as dificuldades dos alunos no processo de aprender diagnosticar, cabendo citação às que se relacionam aos hábitos de estudo, ao aprendizado sobre como manusear corretamente os livros, à deficiência teórica e prática e à aplicação do conhecimento diagnóstico, entre outros.

No que tange às dificuldades de cunho teórico, são dificuldades individuais que demonstram falta de requisitos básicos para desenvolver a atividade diagnóstica, assim como podem resultar do foco na doença mesmo quando se fala de diagnóstico de enfermagem. Fica clara a falta de investimento cognitivo no raciocínio diagnóstico, no processo de pensamento envolvido e operações mentais que são basilares para aprender diagnosticar.

Por outro lado, as informações fragmentadas do DE e desconectadas da realidade de cuidado faz com que os alunos tenham dificuldades em associar teoria e prática dessa atividade. A memória é uma ação mental importante para esta aprendizagem; vale ressaltar que a forma de organizar e armazenar informações, assim como de recuperação, faz parte de um processo de monitoração da aprendizagem, sendo fundamental para a aprendizagem diagnóstica.

Assim, a análise e discussão dos resultados, aqui retratados, mostram que a aprendizagem do diagnóstico tornase difícil. Caberia então investigar se, no processo de ensinar, as informações são suficientes tanto do ponto de vista do conhecimento teórico quanto prático. Ainda mais, averiguar a relação existente entre as aulas teóricas e a prática, identificando se há condições para a vivência do processo e a aprendizagem significativa. Nos casos em que a prática é restrita, haveria dificuldade adicional para o aluno em associar o que o professor ministrou nos conteúdos teóricos com a prática clínica.
Como conclusões sobre as dificuldades de aprendizagem dos alunos pesquisados, podemos afirmar que, na aprendizagem do diagnóstico de enfermagem, os alunos identificam dificuldades relacionadas à estrutura teórica, à aplicação prática de diagnosticar, ao conhecimento e uso das classificações diagnósticas; falta-lhes, também, percepção do raciocínio clínico envolvido e que resulta no diagnóstico.

Essa condição, acreditamos, pode representar que isso se deva pela falta de automonitoramento de seu estado atual de conhecimento, e das metas que deve alcançar com esse conhecimento, assim como do que isso significa para sua prática assistencial.

Assim sendo, todos estes são aspectos que, segundo a literatura, a metacognição pode ajudar. Isto é particularmente importante no caso da medicina, enfermagem e de outros profissionais de saúde. Estes são profissionais que tem como foco solucionar problemas de ordem fisiopatológica e também de relações humanas, situação em que são fundamentais competências intelectuais que incluam habilidades de automonitoramento e regulação do desempenho de situações complexas. ${ }^{15}$

A partir dessa perspectiva, os prováveis benefícios desta pesquisa para a área de fundamentos do cuidado de enfermagem são: a reflexão e discussão pela comunidade acadêmica sobre o processo que envolve a aprendizagem do diagnóstico de enfermagem, a possibilidade de conhecer a estrutura cognitiva do aluno a partir do que ele conhece de si mesmo, e a capacidade de fazer representações que envolvam o processo de aprender; neste caso, de aprender o diagnóstico de enfermagem, que envolve um raciocínio intelectual e processual que é complexo. Além disso, produzir conhecimento sobre processos de aprendizagem em áreas específicas pode subsidiar o professor em seu trabalho diário.

\section{REFERÊNCIAS}

1.NANDA Internacional. Diagnósticos de Enfermagem da NANDA 20092011. Porto Alegre: Artmed; 2010.

2.Flavel JH, Miller PH, Miller SA. Desenvolvimento cognitivo. $3^{\mathrm{a}}$ ed. Porto Alegre:Artmed; 1999.

3. Brandão MAG. Associação entre a interação e a metacognição: características e perspectivas de uma comunidade virtual de enfermagem [dissertação]. Rio de Janeiro (RJ): Núcleo de Tecnologia Educacional, Universidade Federal do Rio de Janeiro; 1999.

4. Almeida MA. Competências e o processo ensino:aprendizagem do diagnóstico de enfermagem: concepções de docentes e discentes [tese]. Porto Alegre: Pontifícia Universidade Católica do Rio Grande do Sul; 2002.

5. Stedile NLR. Intervenção metacognitiva como estratégia de aprendizagem em prevenção de problemas de saúde [tese]. São Paulo: Escola Paulista de Medicina, Universidade Federal de São Paulo; 2002. 
6. Nelson TO, Narens L. Why investigate metacognition? In: Metcalfe J, Shimamura PA. Metacognittion: knowing about knowing. Londres: Bradford Book; 1994. p.1-24.

7. Peixoto MAP, Brandão MAG, Santos G. Metacognição e tecnologia educacional simbólica. Rev Educ Med. 2007; 31(1): 67-80.

8. Zabalza MA. Diários de aulas. Lisboa: Porto Ed; 2004.

9. Minayo MCS. 0 desafio do conhecimento. São Paulo: Hucitec; 2006.

10.Bordenave J. Estratégias de ensino-aprendizagem. $29^{\mathrm{a}}$ ed. Petrópolis(RJ): Vozes; 2008.

11. Carpenito-Moyet ப. Compreensão do processo de enfermagem: mapeamento de conceitos e planejamento do cuidado para estudantes. Porto Alegre: Artmed; 2007.

12. Balduino AFA, Mantovani MF. LacerdaMR. 0 processo de cuidar de enfermagem ao portador de doença crônica cardíaca. Esc Anna Nery. 2009;13(2): 342-51.

13. Hacker DJ, Dunlosky J, Graesser AC. Metacognicion in educational theory and pratice.London: LEA; 1998.

14. Dejano T, Sobral. Características do Inventário de Raciocínio Diagnóstico de Bordage, Grant e Marsden. Psicologia: teoria e pesquisa. 2001 jan/ abr;17(1): 79-85.

15. Quirk M. Intuition and metacognition in medical education: keys to developing expertises. New York: Springer Publishing Company; 2006. 